\title{
Controlled Delayed Aortic Repair in Acute Aortic Syndrome and Multiorgan Failure: An Option in Selected Cases
}

\author{
Daniel S. Dohle ${ }^{1}$ Konstantinos Tsagakis ${ }^{1}$ Saifeldin Ibrahim ${ }^{2}$ Björn Plicht ${ }^{3}$ Heinz Jakob ${ }^{1}$ \\ ${ }^{1}$ Department of Thoracic and Cardiovascular Surgery, University \\ Hospital Essen, West-German Heart Center, Essen, Germany \\ 2 Department of Cardiology, Evangelisches Krankenhaus Oberhausen - \\ EKO, Oberhausen, Germany \\ ${ }^{3}$ Department of Cardiology, University Hospital Essen, West-German \\ Heart Center, Essen, Germany \\ Thorac Cardiovasc Surg Rep 2015;4:52-55. \\ Address for correspondence Dr. med. Daniel S. Dohle, MD, \\ Department of Thoracic and Cardiovascular Surgery, University \\ Hospital Essen, West-German Heart Center Essen, Hufelandstrasse 55, \\ 45147 Essen, Germany (e-mail: ds.dohle@uk-essen.de).
}

\author{
Abstract \\ Keywords \\ - aortic syndromes \\ - multiorgan failure \\ - kidney failure \\ - liver failure
}

The factor time plays an important role in diagnosis and treatment of acute aortic syndromes, not only as a short interval between diagnosis and surgical therapy, but also as a delay of definitive therapy in the selected cases. Severe organ damage caused by malperfusion or other mechanisms can be overcome by a surgical delay, improving prognosis. We report a successful attempt of a controlled delayed surgical treatment in a patient with an acute aortic syndrome complicated from multiorgan failure.

\section{Introduction}

In acute syndromes of the ascending aorta, immediate aortic repair via median sternotomy represents the gold standard of therapy. In critically ill patients with preexisting multiorgan failure, additional trauma of surgery, use of cardiopulmonary bypass, and hypothermia may further harm the patient and increase mortality. According to our experience with delayed surgical aortic repair in acute aortic dissection complicated from severe visceral malperfusion, we report a case of an acute aortic syndrome presenting with already evident multiorgan failure due to cardiac tamponade.

\section{Case Report}

In May 2013, a 50-year-old male patient was admitted to a referring hospital suffering from dyspnea and thoracic pain. Known preexisting conditions were allergic asthma and prostate cancer. The patient had to be put on ventilation because of rapid emerging cardiogenic shock with liver and renal failures. Transesophageal echocardiography (TEE) revealed an aneurysm of the ascending aorta and pericardial effusion, which was relieved by pericardiocentesis $(500 \mathrm{~mL}$ in total). Computed tomographic angiography (CTA) confirmed an aneurysm of the ascending aorta (maximum diameter, $53 \mathrm{~mm}$ ) with a small localized tear in the ascending aorta, pericardial, and pleural effusion (-Fig. 1). The patient was transferred immediately to our department for surgical aortic repair.

According to the Essen concept, the patient was admitted directly to our hybrid operating room (OR). ${ }^{1}$ On admission, the patient was sedated, ventilated, and was hemodynamically stable under moderate adrenergic support. Renal and liver failures and consecutive coagulation disorders were evident by elevated transaminases, creatinine, and anuria (AST, 8,558 U/L; ALT, 5,170 U/L; lactate dehydrogenase [LDH], $5,934 \mathrm{U} / \mathrm{L}$; INR, 2.3 ; creatinine, $3.8 \mathrm{mg} / \mathrm{dL}$ ). TEE showed a good ventricular function, pericardial and pleural effusion, no dissection membrane, and only minimal aortic valve regurgitation. Angiography and intravascular ultrasound confirmed the aneurysm and the small entry tear in the inner curvature of the ascending aorta (- Fig. 1). A concomitant coronary disease was excluded and perfusion of all aortic branches was without pathologic findings. Because of imminent cardiac tamponade sternotomy on the hybrid OR table and staged release of blood, tinged pericardial effusion was performed which improved the hemodynamic situation. In received

September 29, 2014

accepted

November 10, 2014

published online

January 20, 2015
DOI http://dx.doi.org/ 10.1055/s-0034-1396894. ISSN 2194-7635.

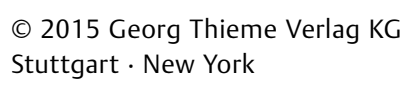

License terms

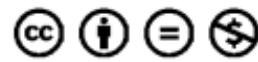




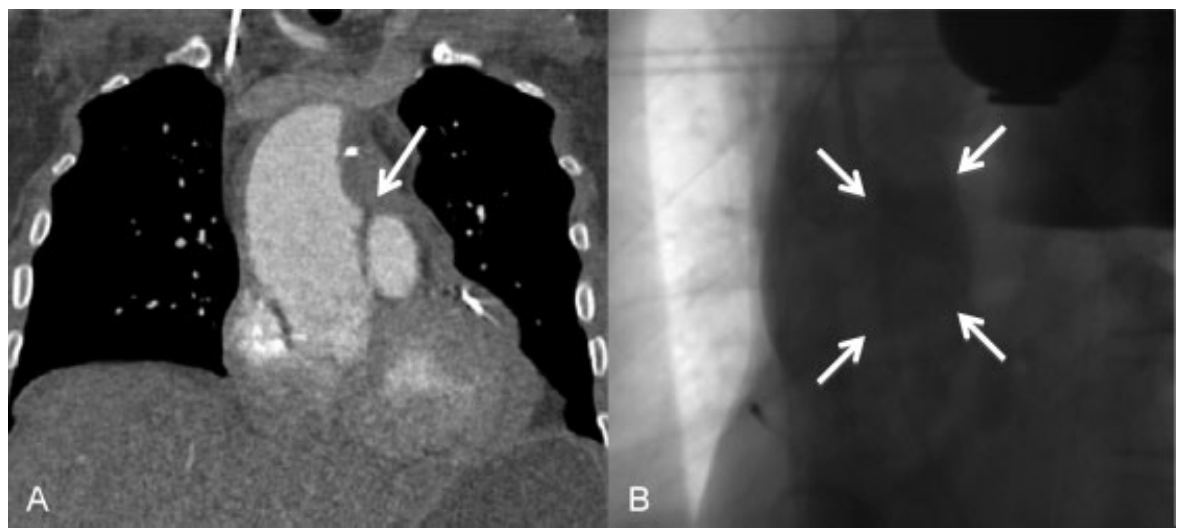

Fig. 1 (A) Preoperative computed tomographic scan and (B) Hybrid room angiography with aneurysm of the ascending aorta, no dissection membrane, only a small tear can be localized toward the pulmonary artery (marked by arrows).

the inner curvature of the aorta, a small circumscribed hematoma indicated the contained rupture. The wall of the residual proximal aorta as well as the arch was normal. To avoid a burdening operation with heart lung machine in this acute phase with multiorgan failure, we decided to delay the operation. Pericardia and pleurae were drained, sternum closed, and the patient admitted sedated and ventilated in our intensive care unit (ICU) with continuous hemodynamic and echocardiographic monitoring without any adrenergics. The blood pressure was strictly controlled (RRsys $<100$ $\mathrm{mm} \mathrm{Hg}$ ) with $\alpha$ - and $\beta$-blockers. During the course, no pericardial effusion was detectable and the mediastinal drainage rate remained under $250 \mathrm{~mL} / 24 \mathrm{~h}$ with serous effusion. Continuous dialysis was initiated because of the acute re nal failure with anuria. Regular liver perfusion was confirmed by ultrasonography. Neurological status was repeatedly controlled during short sedation pauses and CCT showed no pathologies. After further rising of the transaminases and lactate dehydrogenase (AST, 16,463 U/L; ALT, 7,979 U/L; LDH, 11,413 U/L) within 24 hours, these serum markers decreased and liver function recovered (INR, 1.04) within the next 8 days (-Fig. 2 ).

An elective operation was performed on the 8 th day. CPB was instituted via cannulation of the arch at $32^{\circ} \mathrm{C}$. Intra- operatively, a spiral transmural tear was found $2 \mathrm{~cm}$ above the sinotubular junction at the inner curvature toward the pulmonary artery (-Fig. 3 ). The aortic valve was unaltered and the distal ascending aorta not affected. A supracoronary replacement of the ascending aorta with a vascular prosthesis $(30 \mathrm{~mm})$ was performed within 43-minute cross-clamp time and 71-minute bypass time. Because of the delayed and confused awakening and pneumonia, the patient was extubated on the 5th postoperative day.

On the 8th postoperative day, the patient was moved to the ICU of the referring hospital and discharged 2 weeks later after renal recovery and without any impairment. Three months after the initial event, the patient was back at work. In 1-year follow-up, the patient was fully recovered and the CTA showed no further pathologies.

\section{Discussion}

When Deeb et $\mathrm{al}^{2}$ focused on the primary management of malperfusion with nonoperative reperfusion and surgical delay to allow the end-organ failure to resolve and correct metabolic disorders before surgical aortic repair the concept was discussed highly controversial.

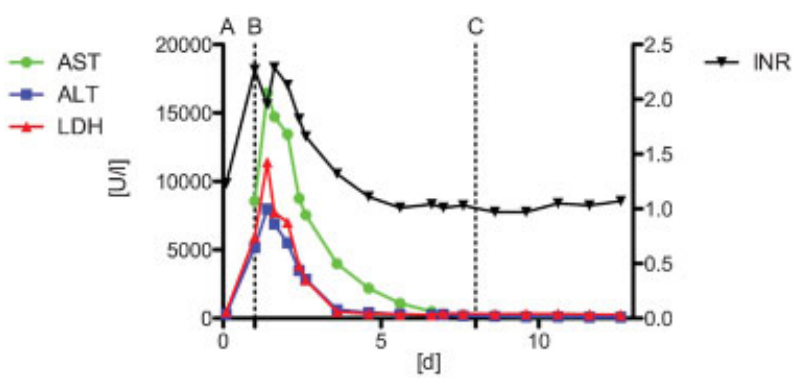

Fig. 2 Time course of the transaminases (AST and ALT), lactate dehydrogenase (LDH) and coagulation (INR). (A) Short after the initial event, ALT and LDH are only minimal increased. (B) At arrival in the hybrid room, the markers are already distinctly elevated and even rise the next day. (C) After recovery over the next days, the markers decrease and definite aortic repair is performed on day 8 .

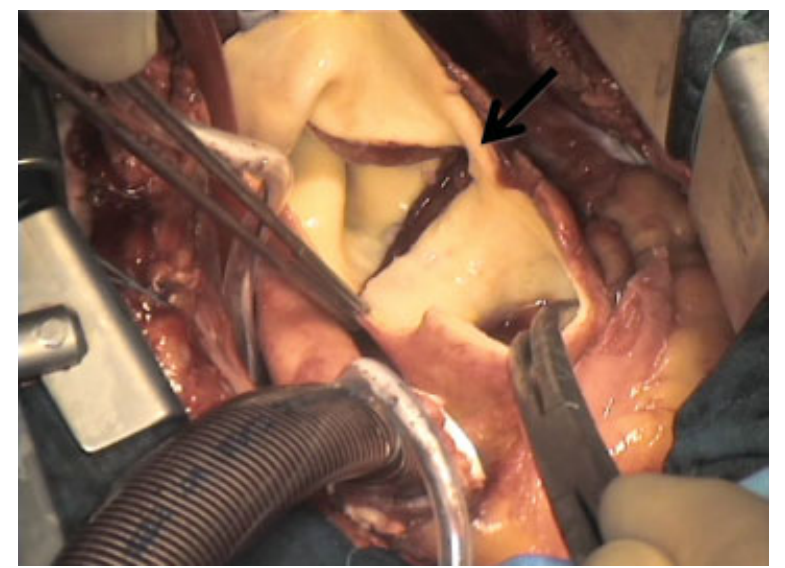

Fig. 3 Intraoperative finding: spiral transmural tear $2 \mathrm{~cm}$ above the sinotubular junction toward the pulmonary artery. 
In the cases of trauma surgery, the principle of "damage control surgery" with the focus being on physiological optimization before repair has led to dramatically improved survival rates and is widely accepted today. ${ }^{3}$ In cardiac surgery, preoperative organ failure is known to increase operative mortality. ${ }^{4}$

An ICU controlled surgical delay after interventional restoration of a more than 6 hours lasting visceral or peripheral malperfusion with clinical signs and elevated serum markers indicating severe organ damage is part of the Essen concept in hemodynamic stable patients without severe aortic valve regurgitation. ${ }^{1}$ Avoiding an additional trauma of surgery, heart-lung machine and hypothermia may help overcome the multiorgan failure. ${ }^{5}$

In the reported case, cardiac tamponade, although patient was immediately relieved by pericardiocentesis in the referring hospital, led to low cardiac output and venous stasis in the liver. On admission to the hybrid OR, the patient already suffered from multiorgan failure with liver and renal failure indicated by high transaminases, coagulation disorder, and anuria. Aortic repair was delayed to avoid an additional liver damage by aortic surgery. Definite aortic repair was performed after 8 days, when decreasing transaminases and rising INR marked liver restoration.

Of course, this approach was only possible due to stable hemodynamic after surgical relieve of the tamponade, no aortic regurgitation, and no intraoperative signs of an imminent rupture. It can only be recommended in selected cases if continuous hemodynamic and echocardiographic monitoring, as well as the capability of immediate surgical intervention are available. In the reported case, the secondary organ damage was diagnosed and individually managed according to a multidisciplinary concept.

\section{References}

1 Tsagakis K, Konorza T, Dohle DS, et al. Hybrid operating room concept for combined diagnostics, intervention and surgery in acute type A dissection. Eur J Cardiothorac Surg 2013;43(2): 397-404

2 Deeb GM, Williams DM, Bolling SF, et al. Surgical delay for acute type A dissection with malperfusion. Ann Thorac Surg 1997;64(6): 1669-1675, discussion 1675-1677

3 Jaunoo SS, Harji DP. Damage control surgery. Int J Surg 2009;7(2): 110-113

4 Thourani VH, Chowdhury R, Gunter RL, et al. The impact of specific preoperative organ dysfunction in patients undergoing aortic valve replacement. Ann Thorac Surg 2013;95(3):838-845

5 Tsagakis K, Pizanis N, Kamler M, et al. ICU controlled delay for acute type a aortic dissection repair after intervention for total visceral malperfusion: a way out of a dilemma? Thorac Cardiovasc Surg 2008;56(5):298-300

\section{Invited Commentary}

\author{
Martin Czerny, MD, MBA, FEBVS \\ Department of Cardiovascular Surgery, University \\ Hospital, Zurich, Switzerland \\ martin.czerny@usz.ch
}

Primary surgical repair of acute type A aortic dissection remains the treatment of choice in the majority of patients when anticipated benefit outweighs risk. Several preoperative factors are known to adversely affect the outcome. Probably, one of the most decisive preoperative conditions is malperfusion, defined as compromised blood flow in one or several end-organs by the dissection process itself or by pericardial effusion. Therefore, different strategies to resolve malperfusion have been developed. The most frequently chosen one is what we would call "quick" establishment of regular flow conditions by primary surgical repair.

However, clinical reality frequently teaches us the hard way that this strategy alone is merely partially successful. As a consequence, pioneering groups have established an alternative to primary surgical repair. This concept focuses on primary restoration of blood flow by interventional means such as stenting or stent-graft placement in various segments of the downstream aorta or by hemodynamic and consequently metabolic optimization with secondary surgical repair after a phase of recompensation-like in this case.
When introducing disruptive concepts, several questions have to be asked:

1. Is this concept per se justified as it is against the accepted standard?

2. Is the potential attrition rate in the time between first and second step acceptable?

3. How long shall the phase of recompensation be calculated in contrast to the risk of secondary aortic rupture?

4. How shall disease progression (pericardial effusion and aortic insufficiency) be reliably monitored during recompensation?

A specific additional question in this case might be why, from a clinical point more or less to be expected, secondary rupture did not occur?

Disruptive concepts in any kind of treatment of cardiovascular pathology have always been and will always be the chance to alter our traditional way of thinking about a natural disease progression or an established treatment modality. The approach presented here should help us accept that natural disease progression might be different from what we think it always is. Furthermore, we should regard such reports proposing a disruptive concept as a unique chance for a subset of patients to be successfully treated in an otherwise potentially unwinnable clinical situation. 


\section{Editor's Commentary: "Malperfusion in Acute Aortic Dissection: A Paradigm Shift?”}

Prof. Dr. Andreas Böning

Klinik für Herz-, Kinderherz- und Gefäßchirurgie, Standort Gießen Universitätsklinikum Gießen und Marburg GmbH 35392 Gießen,

Germany

andreas.boening@chiru.med.uni-giessen.de

Invited commentaries to scientific papers are excellent measures to stimulate a scientific discussion. Invited commentaries to case reports, however, are rare, but follow the same principle: medical issues that are controversially discussed or are developing in a different direction as in the past are often presented as case reports needing an annotation.

This is also true for this case report, which describes a defined therapy pathway for patients with acute aortic dissection type A (AADA) presenting with malperfusion syndromes. These patients are at the highest risk of mortality and morbidity among all AADA cases.

Would it be possible to decrease these risks if the malperfusion were treated first instead of replacing the ascending aorta?
Is it advisable to treat malperfusion first, although this would deviate from the actual treating principles or guidelines?

A recent report ${ }^{1}$ could show a decreased risk of AADA patients with malperfusion when the malperfusion was treated first instead of the ascending aorta. As so often in surgery, it is hardly likely that this exciting question can ever be examined within a prospective trial.

Is stenting of the AADA therefore a bailout procedure, or is it a valuable addition for patients with malperfusion?

As you see, there are a lot of questions accompanying such a paradigm shift in AADA therapy. Therefore, the invited commentary by Czerny to this case report is intended to stimulate the scientific discussion on this important topic.

\section{Reference}

1 Perera NK, Galvin SD, Seevanayagam S, Matalanis G. Optimal management of acute type A aortic dissection with mesenteric malperfusion. Interact Cardiovasc Thorac Surg 2014;19(2): 290-294 